\title{
Inadvertent arterial insertion of a central venous catheter: delayed recognition with abrupt changes in pressure waveform during surgery -A case report-
}

\author{
Yong Seon Choi ${ }^{1,2}$, Ji Young Park ${ }^{1}$, Young Lan Kwak ${ }^{1,2}$, and Jong Wha Lee \\ ${ }^{1}$ Department of Anesthesiology and Pain Medicine and ${ }^{2}$ Anesthesia and Pain Research Institute, College of Medicine, Yonsei \\ University, Seoul, Korea
}

We present a case of inadvertent arterial insertion of a central venous catheter, identified during a pericardiectomy procedure after observing abrupt changes in pressure waveform and confirmed via arterial blood gas analysis and transesophageal echocardiography. Central venous pressure measurement was initially $20 \mathrm{mmHg}$ in supine, and then elevated to $30-40 \mathrm{mmHg}$ in right lateral decubitus, presumably resulting from constrictive physiology of pericarditis. The pressure waveforms, however, abruptly changed from a venous to an arterial waveform during surgery. When visual discrimination between arterial and venous blood regurgitation is unreliable, anesthesiologists should confirm that using all the available methods one has on the scene, especially after at least two unsuccessful attempts or in patients with advanced age or clinical conditions resulting in jugular venous dilation. To prevent arterial catheterization, one should limit the leftward rotation of the head by $\left\langle 40^{\circ}\right.$ and consider using ultrasoundguided method after more than two unsuccessful attempts. (Korean J Anesthesiol 2011; 60: 47-51)

Key Words: Arterial insertion, Central venous catheterization, Intraoperative complication.

\begin{abstract}
Central venous catheterization (CVC) is a standard anesthetic procedure for cardiovascular medications, volume status monitoring, and rapid infusion of fluids and blood products. Among the various approaches possible for CVC, the internal jugular vein is the most preferred site because it has certain advantages including easier catheterization, resultant high
\end{abstract}

rate of success when using only anatomical landmarks of the sternocleidomastoid muscle (SCM), and a straighter path to the superior vena cava compared to that of the subclavian vein, resulting in a fewer incidence of malpositioning and more precise measurement of central venous pressure [1]. In contrast, arterial puncture, the most frequent complication of CVC, is

Received: July 29, 2010. Revised: September 8, 2010. Accepted: September 12, 2010.

Corresponding author: Jong Wha Lee, M.D., Department of Anesthesiology and Pain Medicine, and Anesthesia and Pain Research Institute, College of Medicine, Yonsei University, 134, Shinchon-dong, Seodaemun-gu, Seoul 120-752, Korea. Tel: 82-2-2228-8516, Fax: 82-2-364-2951, E-mail: jhanes@yuhs.ac

(c) This is an open-access article distributed under the terms of the Creative Commons Attribution Non-Commercial License (http:// creativecommons.org/licenses/by-nc/3.0/), which permits unrestricted non-commercial use, distribution, and reproduction in any medium, provided the original work is properly cited. 
associated more with an internal jugular approach than with a subclavian approach, the incidence of which is reportedly $3-9 \%$ [1-4]. In most cases of CVC, one can differentiate a successful venous puncture from an arterial puncture based on the color and pulsation of regurgitated blood flow. However, these findings may be unreliable depending on oxygen saturation, systemic blood pressure, and tricuspid regurgitation. Therefore, when visual discrimination is not appropriate, venous puncture must be confirmed using pressure waveforms $[5,6]$.

We report a case of inadvertent arterial insertion of a central venous catheter, identified during a pericardiectomy procedure after observing abrupt changes in pressure waveform, and confirmed via arterial blood gas analysis and TEE. A review of the literature is also presented.

\section{Case Report}

A 64-year-old man, weighing $47 \mathrm{~kg}$ and having a height of $154 \mathrm{~cm}$, was scheduled for a pericardiectomy for treatment of constrictive pericarditis. When he was hospitalized with a two-week history of aggravating dyspnea, he had been placed on outpatient follow-up for pulmonary tuberculosis, previously diagnosed with dyspnea on exertion and pleural effusion. His past medical history included diabetes mellitus diagnosed 20 years ago and stent insertion in the left anterior descending (LAD) coronary artery eight years ago. Preoperative ECG revealed left atrial enlargement, and ST depression and flat T-wave on lead II, III and $\mathrm{V}_{4-6}$. Chest radiograph showed pulmonary edema and bilateral pleural effusion. Coronary computerized tomography demonstrated that the LAD stent was intact, but there was $>50 \%$ narrowing of the left circumflex coronary artery, and pericardial thickening with adhesion to the right atrial and left ventricular lateral walls. Echocardiography showed a left ventricular ejection fraction of $73 \%$, and mild pulmonary hypertension with a systolic right ventricular pressure of $38 \mathrm{mmHg}$.

An hour before surgery, premedication was done with IM morphine $3 \mathrm{mg}$. On arrival, continuous monitoring of ECG lead II and $\mathrm{V}_{5}$ was initiated, and the radial artery cannulation was done under local anesthesia for pressure monitoring. Vital signs before induction of anesthesia were HR $85 / \mathrm{min}$, ABP $108 / 68 \mathrm{mmHg}$, and $\mathrm{SpO}_{2} 98 \%$. Anesthesia was induced with IV midazolam $3 \mathrm{mg}$ and ketamine $20 \mathrm{mg}$. Rocuronium $50 \mathrm{mg}$ was administered to facilitate endobronchial intubation with a 35 Fr left-sided double-lumen tube. After intubation, lungs were ventilated with a tidal volume of $8-10 \mathrm{ml} / \mathrm{kg}$, adjusted to maintain end-tidal $\mathrm{CO}_{2}$ within $33-38 \mathrm{mmHg}$. Anesthesia was maintained with $1.0-1.5 \%$ sevoflurane in $60 \%$ oxygen in air and continuous infusion of remifentanil at $0.1-0.3 \mu \mathrm{g} / \mathrm{kg} / \mathrm{min}$.

After induction of anesthesia, the patient was turned to the head-down position with the head rotated leftward by approximately $60^{\circ}$ to facilitate internal jugular catheterization. An 18-G puncture needle was inserted at the conjoined site of both bellies of the SCM, and was advanced toward the ipsilateral nipple until free regurgitation of venous, non-pulsatile blood flow was observed. During the first attempt, the puncture needle had to be withdrawn, as the J-tipped guide wire could be advanced $<10 \mathrm{~cm}$. After $5 \mathrm{~min}$ of manual compression, jugular venous puncture was retried in the same manner, and nonpulsatile, free-regurgitant blood flow was again obtained. In the second attempt, the J-tipped guide wire was inserted into the vessel without significant resistance, even though it was initially difficult to advance the wire and the puncture needle had to be withdrawn a few millimeters to obtain free regurgitant blood flow. Then, a 7-Fr double-lumen central venous catheter (Arrow Gard Blue, Arrow International, Inc., USA) was inserted using the Seldinger method and was suture-fixed at $16 \mathrm{~cm}$ in the skin after confirming free inflow of fluid. At the end of procedure, the guide wire withdrawn showed no deformations. Central venous pressure (CVP) was measured to be $20 \mathrm{mmHg}$ with the patient in a supine position and thereafter was maintained within 30-40 mmHg in the right semi-lateral decubitus position at approximately $30^{\circ}$ during one-lung ventilation for surgery. Such a high CVP was considered to be related to the constrictive pericarditis, as the pressure measurement was less than the mean arterial pressure of the radial artery $(80-85 \mathrm{mmHg})$ and the waveform was flat without pulsations, being affected by right-side one-ling ventilation and surgical manipulation of the heart. During the catheterization, vital signs were stable with HR 83/min, BP 90-100/65-68 $\mathrm{mmHg}$, and $\mathrm{SpO}_{2} 99$ $100 \%$. Arterial blood gas analysis was done after induction of anesthesia and revealed $\mathrm{pH}$ 7.486, $\mathrm{PaO}_{2} 331 \mathrm{mmHg}$, and $\mathrm{PaCO}_{2}$ $36.2 \mathrm{mmHg}$ with $\mathrm{FiO}_{2}$ 0.6.

Approximately 50 minutes after the surgery began, the pressure waveform from the central venous catheter changed abruptly into what was seemingly an arterial waveform, with a mean pressure of $61 \mathrm{mmHg}$, almost same to the mean arterial pressure of $62 \mathrm{mmHg}$ (Fig. 1). Simultaneously, blood regurgitated through the fluid line connected to the central venous catheter. Even though about $300 \mathrm{ml}$ of fluid had already been administered, no hematoma or edema was shown in the puncture site. Arterial blood gas measurement was done on blood withdrawn from the central venous catheter, indicating a $\mathrm{PaO}_{2}$ of $438.7 \mathrm{mmHg}$ with a $\mathrm{FiO}_{2}$ of 0.6 , confirming that it was arterial blood. Furthermore, the catheter was apparent in the aortic arch on TEE (Fig. 2). Fluid infusion was immediately stopped, and the catheter was removed when the surgery was completed. After confirming that there was no bleeding or developing hematoma in the puncture site with $>15 \mathrm{~min}$ of compression, the double-lumen tube was replaced with a 


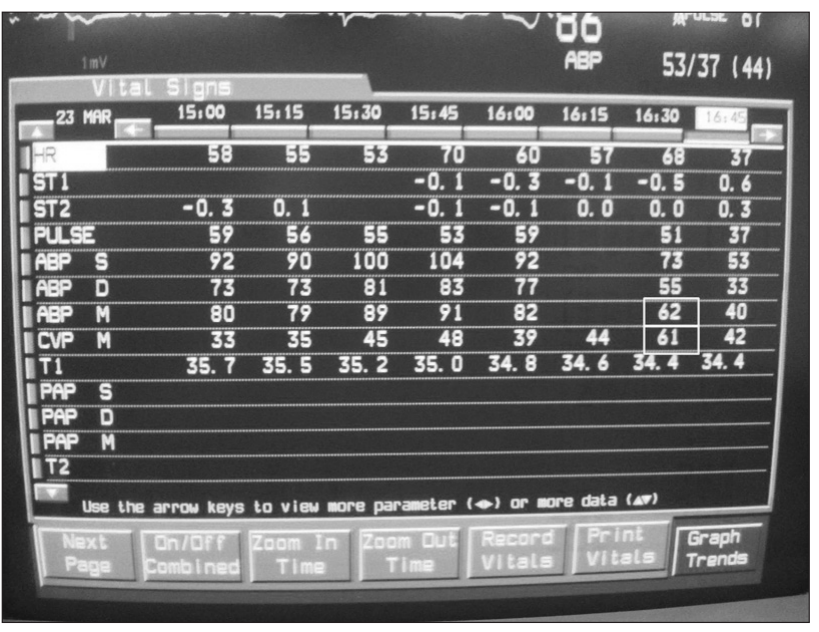

Fig. 1. Photograph shows images of the patient monitor, showing the tabular presentation of serial changes in hemodynamic parameters. Numbers in rectangles indicates the same values of mean arterial pressure and central venous pressure.

single-lumen endotracheal tube. Thereafter, the patient was transferred to the intensive care unit with the endotracheal tube in situ. Extubation was done after seven days of mechanical ventilatory care for tachypnea and $\mathrm{CO}_{2}$ retention, and the patient was transferred to the general ward without central venous catheter-related complications on the 10th postoperative day.

\section{Discussion}

Most of CVC-related complications are due to vessel trauma. Arterial puncture is most frequently encountered, but rarely results in more serious complications, such as hematoma development compromising airway, thromboembolism causing cerebrovascular accident, arteriovenous fistula or pseudoaneurysm $[3,7,8]$. Arterial catheterization can happen after arterial puncture, but the incidence is very low $(<0.1 \%)$ [9]. Obesity, hypotension, reduced hemoglobin, low oxygen saturation or unavailability of ultrasonic guidance are risk factors for arterial catheterization [2,3]. In healthy patients, arterial puncture is easily recognized by pulsatile regurgitation of bright red blood; however, in patients with hypoxemia or hypotension, those visual parameters would be unreliable due to a weak pulsation of dark red blood [3]. In contrast, patients with increased CVP, including those with congestive heart failure, tricuspid regurgitation, or constrictive pericarditis, may present with forced venous regurgitation, which can be misinterpreted as arterial puncture. In this case, clinically significant pitfalls on differentiation of arterial puncture are the features of the regurgitant blood flow, including color, pulsation, force, or velocity. As $\mathrm{FiO}_{2}$ had been maintained at 0.6

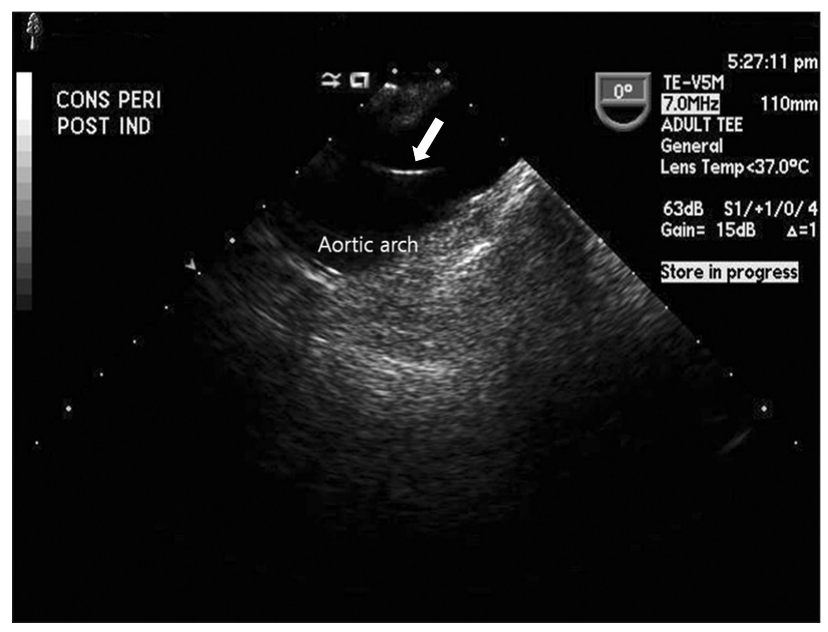

Fig. 2. Echocardiograph shows that a central venous catheter (arrow) is inserted into the aorta.

after induction of anesthesia, it was difficult to macroscopically discriminate between arterial and venous blood. Also, we already anticipated increased blood regurgitation due to the elevated diastolic pressure caused by restrictive physiology of pericarditis. It would be highly suspicious of an arterial puncture if massive regurgitation of bright red blood with pulsation or large hematoma was observed; however, in this case, those findings were not definitive. Moreover, fluid was running well when the catheter was initially indwelled. Altogether, these findings interfered with the early recognition of arterial catheterization.

Risk factors of arterial puncture that affected this case are as follows. First, overlap between the carotid artery and jugular vein could be increased with leftward head rotation. Together with a relatively deep insertion of the puncture needle, such an increased overlapping could facilitate a penetration of the anterior wall of the carotid artery through the jugular vein. Troianos et al. [10] demonstrated that when the head was maximally rotated leftward without discomfort, an overlap of $>75 \%$ was observed in $54 \%$ of patients, especially in those with advanced age ( $>60 \mathrm{yr}$ ) and venous dilation. Kim et al. [11] also found that the degree of overlap between the carotid artery and jugular vein increased with leftward head rotation in Korean patients. Considering the small build of our patient, puncture needle could be inserted relatively deep, and eventually penetrate the anterior wall of the carotid artery after advanced through the jugular vein. And non-deformed guide wire withdrawn could be another evidence of carotid puncture, not subclavian artery. Second, multiple attempts at venous puncture are known to increase the incidence of mechanical complications of CVC, reportedly increasing by about 10 -fold after at least two unsuccessful attempts even with 
an experienced physician. The ultrasound-guided method is reported to be helpful to reduce the complications $[2,12]$.

It is unclear why the pressure waveform abruptly changed $1 \mathrm{hr}$ after the arterial catheterization. Non-pulsatile, plain waveform, mimicking venous property despite arterial insertion, might be attributed to kinking of the catheter or partial obstruction of the catheter tip against the vessel wall. Some measurement errors in pressure transducer system, such as excessive damping, could result in such a plain arterial waveform. Abrupt change in pressure waveform and measurement would be attributed to a sudden release of previously kinked catheter or position shift of catheter due to surgical manipulation of the heart. Hwang and Kim [13] reported a case of gradual increase in CVP from 34-37 $\mathrm{mmHg}$ when the catheter was indwelled, to almost the same to the mean arterial pressure. Similar to our case, they confirmed arterial insertion via arterial blood gas measurement because the pressure waveform did not appear to be arterial and they assumed damping to be a primary cause. They accomplished the catheterization at the first attempt, and presented no difficulties during catheterization. Proper discrimination between the unmasked arterial and central venous waveforms could be difficult due to atrial fibrillation, which presumably resulted from hypotension during induction in their patient with severe coronary artery disease. Unlike their case, we observed the abrupt change of previously plain waveform to arterial waveform (Fig. 3), requiring no comparison of arterial gas measurement with venous blood.

Recently, percutaneous/intravascular intervention with a hemostatic device was shown to be safe and applicable treatment for arterial trauma [14]. In this case, however, the catheter was removed and then manually compressed. This can sometimes cause bleeding, cerebrovascular accident or

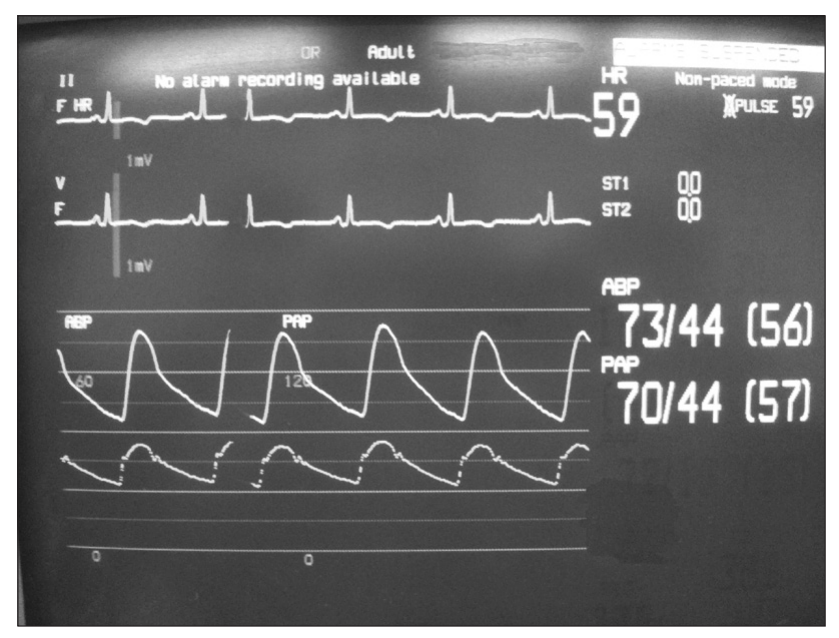

Fig. 3. Photograph shows images of the patient monitor, showing identical arterial waveforms and pressure measurements from radial artery ("ABP") and central venous catheter ("PAP"). pseudoaneurysm, the risk of which, however, is dependent on the catheter size, elapsed time from catheterization, and the site of catheterization $[3,14]$. Because the arterial catheterization was maintained briefly, no hematoma formation in the aorta was confirmed with TEE, and the internal jugular vein could be compressed more easily than the subclavian vein, the removal and compression were judged to be appropriate. The concern was the possibility of surgical field contamination; the removal and compression were done after the surgery was finished.

When visual discrimination between arterial and venous blood regurgitation is unreliable, anesthesiologists should confirm where one punctures using all the available methods one has on the scene, especially after at least two unsuccessful attempts or in patients with advanced age or clinical conditions resulting in jugular venous dilation. To prevent arterial catheterization, one should limit the leftward rotation of the head by $<40^{\circ}$ and consider using ultrasound-guided method after more than two unsuccessful attempts.

\section{References}

1. Ruesch S, Walder B, Tramèr MR. Complications of central venous catheters: internal jugular versus subclavian access--a systematic review. Crit Care Med 2002; 30: 454-60.

2. Schummer W, Schummer C, Rose N, Niesen WD, Sakka SG. Mechanical complications and malpositions of central venous cannulations by experienced operators. A prospective study of 1794 catheterizations in critically ill patients. Intensive Care Med 2007; 33: 1055-9.

3. Pikwer A, Acosta S, Kölbel T, Malina M, Sonesson B, Akeson J. Management of inadvertent arterial catheterisation associated with central venous access procedures. Eur J Vasc Endovasc Surg 2009; 38: 707-14.

4. McGee DC, Gould MK. Preventing complications of central venous catheterization. N Engl J Med 2003; 348: 1123-33.

5. Ricci M, Puente AO, Gusmano F, LaRaja R. Central venous access: accidental arterial puncture in a patient with right-sided aortic arch. Crit Care Med 1999; 27: 1025-6.

6. Aghdami A, Ellis R. High oxygen saturation does not always indicate arterial placement of catheter during internal jugular venous cannulation. Anesthesiology 1985; 62: 372-3.

7. Ezri T, Szmuk P, Cohen Y, Simon D, Mavor E, Katz J, et al. Carotid artery-internal jugular vein fistula: A complication of internal jugular vein catheterization. J Cardiothorac Vasc Anesth 2001; 15: 231-2.

8. Droll KP, Lossing AG. Carotid-jugular arteriovenous fistula: case report of an iatrogenic complication following internal jugular vein catheterization. J Clin Anesth 2004; 16: 127-9.

9. Golden LR. Incidence and management of large-bore introducer sheath puncture of the carotid artery. J Cardiothorac Vasc Anesth 1995; 9: 425-8.

10. Troianos CA, Kuwik RJ, Pasqual JR, Lim AJ, Odasso DP. Internal jugular vein and carotid artery anatomic relation as determined by ultrasonography. Anesthesiology 1996; 85: 43-8. 
11. Kim SI, Kang JH, Baek Y, Kim SH, Ok S, Kim SC. The influence of head rotation on the anatomical relationship of the right internal jugular vein and the carotid artery. Korean J Anesthesiol 2008; 55: 538-42.

12. Ruth MJ, Scott DH. Ultrasound guidance for internal jugular vein catheterization. J Cardiothorac Vasc Anesth 2001; 15: 803-4.

13. Hwang WG, Kim GH. Arterial puncture unrecognized by pressure waveform monitoring after central venous catheterization. Korean J Anesthesiol 2004; 46: 363-6.

14. Guilbert MC, Elkouri S, Bracco D, Corriveau MM, Beaudoin N, Dubois MJ, et al. Arterial trauma during central venous catheter insertion: Case series, review and proposed algorithm. J Vasc Surg 2008; 48: 918-25. 